\title{
Combattants saoudiens en Irak : modes de radicalisation et de recrutement
}

Saudis in Iraq: Patterns of Radicalization and Recruitment

\section{Thomas Hegghammer}

Traducteur : Christian Olsson

\section{(2) OpenEdition Journals}

\section{Édition électronique}

URL : http://journals.openedition.org/conflits/2128

DOI : $10.4000 /$ conflits. 2128

ISSN : $1777-5345$

\section{Éditeur :}

CCLS - Centre d'études sur les conflits lilberté et sécurité, L'Harmattan

\section{Édition imprimée}

Date de publication : 20 décembre 2006

Pagination : 111-126

ISBN : 978-2-296-02667-4

ISSN : $1157-996 X$

\section{Référence électronique}

Thomas Hegghammer, « Combattants saoudiens en Irak : modes de radicalisation et de recrutement », Cultures \& Conflits [En ligne], 64 | hiver 2006, mis en ligne le 05 mars 2007, consulté le 30 mars 2021. URL : http://journals.openedition.org/conflits/2128; DOI : https://doi.org/10.4000/ conflits. 2128

Ce document a été généré automatiquement le 30 mars 2021.

Creative Commons License 


\title{
Combattants saoudiens en Irak : modes de radicalisation et de recrutement
}

\author{
Saudis in Iraq: Patterns of Radicalization and Recruitment
}

\author{
Thomas Hegghammer
}

Traduction : Christian Olsson

\section{NOTE DE L'ÉDITEUR}

Article publié en "résonance" au n63 Mort volontaire combattante

Dans le dernier numéro de Cultures \& Conflits, huit auteurs ont exploré le phénomène complexe de la mort volontaire au travers de différentes approches, insistant toutes sur la nécessité de démystifier, de "désislamiser " la notion de suicide combattant et de reconnaître la multiplicité des processus conduisant au sacrifice de soi à l'âge moderne ${ }^{1}$. Le cas de l'Irak, qui est l'arène principale - du moins en termes quantitatifs - de la mort volontaire et du sacrifice de soi aujourd'hui, n'est pourtant pas exploré dans le débat du dernier numéro. Cet article analysera le phénomène des combattants étrangers en Irak, qui se distinguent de beaucoup d'autres combattants suicidaires par le fait suivant: le pays pour lequel ils se proposent de mourir n'est pas leur pays d'origine.

2 Afin de comprendre la configuration immensément complexe et évolutive de l'insurrection en Irak, la plupart des commentateurs ont tendance à établir une distinction stricte entre les combattants étrangers d'un côté et les nationalistes irakiens de l'autre. Les étrangers sont généralement considérés comme les éléments les moins légitimes et les plus extrêmes de l'insurrection. On a beaucoup écrit sur leurs actions en Irak, alors que nous ne savons en réalité que peu de choses sur leurs antécédents, milieux d'origine et motivations ${ }^{2}$. Il semble par conséquent que, dans ce cas précis, l'appel de Bruno Etienne en faveur d'efforts supplémentaires pour 
comprendre l'inacceptable n'a pas été entendu. Nous regarderons ici de plus près l'un des "contingents" étrangers les plus grands et les moins compris en Irak, celui des Saoudiens. Qui sont les combattants saoudiens? Pourquoi quittent-ils une vie paisible de confort matériel pour la guerre et une mort probable dans un pays étranger?

Le militant saoudien est souvent décrit de manière caricaturale comme le "kamikaze » archétypique, l'extrémiste incurable qui recherche la guerre sans aucune autre raison apparente que celle de tuer et d'être tué. Un large pan de la littérature sur l'islamisme militant présuppose l'existence d'un «extrémisme saoudien » qui garantirait un flux permanent de militants vers des zones de conflits et des groupes terroristes. Ce faisant, la question fondamentale de la raison qui pousse certains Saoudiens et pas d'autres à partir pour l'Irak reste ignorée. Ces points de vue semblent aussi faire abstraction de l'individualité et de l'humanité des militants saoudiens. Louis-Jean Duclos, Olivier Grojean et Eleanor Pavey ont montré qu'au sein d'un seul et même mouvement, des voies multiples et plurielles peuvent mener au sacrifice de soi. Pourquoi en irait-il différemment pour les Saoudiens? Afin de répondre à ces questions, il nous faudra nous plonger dans les histoires et trajectoires individuelles et étudier les motivations sous-jacentes ainsi que les modalités de recrutement.

Jusqu'à récemment, ce type de recherche était rendu difficile par la nature clandestine des groupes violents et les règles très strictes d'octroi du visa saoudien. Avec l'avènement $d$ 'Internet - que les militants saoudiens et irakiens ont chaleureusement accueilli - il est devenu possible d'étudier ces communautés au travers de leurs propres écrits et publications. L'Arabie Saoudite a aussi commencé à ouvrir ses portes aux chercheurs occidentaux en sciences sociales et nous a ainsi permis de conduire une vaste recherche de terrain dans le Royaume. En combinant la littérature djihadiste disponible sur Internet et des sources secondaires, nous avons compilé et sérié les noms et biographies de 205 Saoudiens morts en Irak entre 2003 et $2005^{3}$. Nous nous servirons ici de ces informations pour aborder quatre questions : Qui part pour l'Irak ? Pourquoi partent-ils ? Comment partent-ils ? Quel est leur rôle dans l'insurrection?

5 Nous verrons que le nombre exact de Saoudiens partis en Irak est contesté, mais il semblerait qu'il ne dépasse pas 1500 . Ces volontaires sont issus d'une grande diversité de milieux sociaux et de zones géographiques, même si le Nord du Royaume est surreprésenté par rapport au Sud. Ces Saoudiens sont motivés par un nationalisme panislamique et inspirés par des amis ou des membres de leur famille partis avant eux. Certains sont enrôlés par des recruteurs professionnels, d'autres recherchent des «facilitateurs » de leur propre chef. En Irak, les Saoudiens sont surreprésentés parmi les auteurs d'attentats-suicides. Pourtant, le nombre de Saoudiens partis en Irak a connu son apogée à la fin de l'année 2004 et continue de décliner depuis. En effet, les autorités saoudiennes ont pris des mesures pour juguler le recrutement. Ils se heurtent cependant à des résistances politiques internes, du fait des perceptions du phénomène comme participant d'une résistance irakienne légitime.

6 L'implication saoudienne dans des conflits extérieurs n'est pas chose nouvelle. Les Saoudiens morts au combat en Irak ne sont que la dernière génération de ce qui est décrit dans la littérature djihadiste comme la " caravane des martyrs ». Depuis le milieu des années 1980, des milliers de jeunes hommes saoudiens ont combattu en tant que moudjahiddin dans divers pays : l'Afghanistan, la Bosnie-Herzégovine, le Tadjikistan, la Tchétchénie et l'Afghanistan encore une fois ${ }^{4}$. Les Saoudiens n'ont jamais été le seul «contingent» étranger dans ces conflits et n'ont pas toujours été surreprésentés. 
Néanmoins, il existe une représentation commune - alors qu'on ne dispose pas de chiffres incontestables - selon laquelle la participation saoudienne dans des zones de djihad aurait toujours été très élevée 5 .

7 Les raisons de cet activisme ne sont pas à chercher dans la tradition religieuse du wahabisme en soi, ni dans un quelconque extrémisme inhérent à la culture saoudienne, mais plutôt dans la force, en Arabie Saoudite, de ce qu'il est convenu d'appeler le «nationalisme pan-islamique». Ce nationalisme a parfaitement été illustré dans un entretien avec un étudiant saoudien paru dans le Washington Post en 2005 :

«Les Américains ne peuvent pas s'imaginer comment un jeune homme menant un vie décente à Riyad pourrait sentir un tel amour et une telle passion pour un coreligionnaire musulman et se sentir obligé de partir combattre quand il voit des images télévisées d'Irakiens et d'Afghans qui sont tués et torturés. Mais c'est là le résultat de la force des liens de sang islamiques ${ }^{6} »$.

Ces "liens de sang" sont bien sûr imaginaires et trouvent leur origine dans une croyance en l'unité inhérente à la nation musulmane (l'» Oumma»). Comme tous les nationalismes, le pan-islamisme est une construction. Mais pourquoi les Saoudiens auraient-ils un sens plus profond de la solidarité pan-islamique que les musulmans d'autres pays?

9 Les raisons en sont historiques et politiques. Dans les années 1960, le roi Faisal a mis l'accent sur la solidarité musulmane (al-tadamun al-islami) afin de faire contrepoids au nationalisme arabe de Nasser. A partir de la moitié des années 1980, l'Etat saoudien a promu le nationalisme pan-islamique afin d'asseoir sa propre légitimité et de faciliter la mobilisation pour le djihad en Afghanistan. Ce discours nationaliste pan-islamique a accordé une grande importance à la responsabilité morale des Saoudiens dans le soutien aux musulmans opprimés à l'étranger. Au début des années 1990, l'opposition islamiste modérée a commencé à adopter une rhétorique similaire, contestant ainsi l'emprise de l'Etat sur le nationalisme pan-islamique en tant que source de légitimité. A ainsi émergé une culture politique d'affichage concurrentiel de solidarité déclarée avec les causes musulmanes à l'extérieur. Cela a suscité un abaissement du seuil de tolérance $\mathrm{du}$ financement et du soutien militaire aux mouvements de résistance musulmans en des lieux tels que la Palestine, la Bosnie-Herzégovine et la Tchétchénie. La frontière, la ligne entre charité et soutien militaire, a été brouillée et la participation au djihad en est venue à être perçue comme un acte d'altruisme et de courage. Participer à des luttes de résistance territoriales clairement définies - par opposition au terrorisme international ou à la contestation en interne - était considéré par de nombreux Saoudiens religieux comme parfaitement légitime.

Dans la littérature universitaire occidentale, la notion de nationalisme pan-islamique est très rarement utilisée. Ce fait surprenant pourrait provenir de la tendance, dans les pays occidentaux, à associer nationalisme et Etat-nation. Une autre raison pourrait en être le fait que les djihadistes mondialisés sont considérés comme les acteurs les plus extrêmes - et, par conséquent, les moins rationnels - dans le paysage des islamistes militants. Cependant, analyser le djihadisme mondialisé comme une forme extrême de nationalisme pan-islamique permettrait d'expliquer des éléments cruciaux de l'activisme des militants généralement assimilés à al-Qaïda. Ainsi, cela montrerait que, pour les Saoudiens qui vont en Irak, cela ne signifie pas partir à l'étranger pour se battre pour le territoire de quelqu'un d'autre, mais simplement se déplacer vers une autre partie du monde musulman pour défendre le territoire musulman commun. 
11 La situation en Irak après l'invasion menée par les Etats-Unis en mars 2003 était considérée par beaucoup comme un cas d'école de djihad défensif. Des forces militaires étrangères ont occupé un territoire musulman, après ce qui a été largement perçu comme une agression injustifiée. Pour les nationalistes pan-islamiques, il n'y a pas véritablement eu de différence entre la situation en Irak et, par exemple, celle de l'Afghanistan dans les années 1980. Comme le dit un membre des services de renseignements saoudiens :

« Nous avons encouragé nos jeunes hommes à aller combattre pour l'Islam en Afghanistan. Nous avons encouragé nos jeunes hommes à se battre pour l'Islam en Bosnie et en Tchétchénie. Nous avons encouragé nos jeunes hommes à se battre pour l'Islam en Palestine. Maintenant nous leur disons : "vous n'avez pas le droit de vous battre pour l'Islam en Irak" et ils sont confus ${ }^{7} \mathrm{M}$.

12 Ce qui a changé est bien sûr le contexte politique. Dans ladite "guerre contre le terrorisme ", la distinction entre guérilla locale et terrorisme international est devenue insignifiante. De plus, la campagne de violence lancée en Arabie Saoudite en 2003 par des vétérans de l'Afghanistan a contribué à sensibiliser les autorités saoudiennes sur l'acuité du risque représenté par les vétérans. Ainsi des clercs affiliés au gouvernement comme Abd al-Muhsin al-Ubaykan ont déclaré que l'insurrection en Irak constituerait une fitna (sédition) et serait illégitime ${ }^{8}$.

13 Ce changement de climat politique a été parfaitement illustré en novembre 2004, lorsqu'un groupe d'éminents Oulémas saoudiens a publié une déclaration selon laquelle la résistance armée à l'occupation américaine était légitime pour le peuple irakien 9 . Celle-ci n'a pas encouragé les Saoudiens à se rendre en Irak et, dans des déclarations ultérieures, certains signataires ont même découragé explicitement les jeunes Saoudiens de se joindre au djihad irakien ${ }^{10}$. Pourtant, cette déclaration a été largement condamnée par les médias occidentaux et les médias officiels saoudiens, comme une incitation au terrorisme, alors que la déclaration ne faisait que de refléter la position dominante dans les cercles islamistes en Arabie Saoudite. Selon ce point de vue, la résistance irakienne constitue un djihad légitime et représente un devoir collectif (fard kifaya) pour la nation islamique. Il serait ainsi permis - mais pas nécessaire - aux Saoudiens d'y participer ${ }^{11}$. Il va de soi qu'au sein de communautés islamistes plus radicales, la participation au djihad irakien est considérée comme un devoir individuel (fard ayn) pour tous les musulmans, pas seulement pour les Irakiens. La nécessité de résister aux forces de la coalition menée par les Etats-Unis en Irak est ainsi devenue un thème central de la littérature djihadiste dès 2002 et l'Irak, la cause pan-islamique par excellence ${ }^{12}$. En effet, au sein d'une Arabie Saoudite pan-islamique, la situation de ce pays a touché certains au plus profond d'eux-mêmes.

14 Les premiers Saoudiens sont arrivés au Nord de l'Irak au début de l'année 2003, avant l'invasion menée par les Etats-Unis ${ }^{13}$. Depuis le mois de mars de la même année, les médias ont rapporté que des combattants étrangers, comprenant des Saoudiens, entraient en Irak ${ }^{14}$. Les premières arrestations de Saoudiens sont survenues en juin 2003 et des sites Internet djihadistes ont commencé à annoncer la mort de « martyrs » saoudiens en Irak durant l'été ${ }^{15}$.

15 Dans les biographies djihadistes, les dates de départ indiquent que le flux de recrues saoudiennes vers l'Irak a considérablement augmenté à l'été 2004 et a connu son apogée en novembre 2004. L'augmentation, dans la seconde moitié de cette même année, peut s'expliquer par différents facteurs. En Arabie Saoudite, la campagne de 
terrorisme interne a été très affaiblie au milieu de l'année 2004, et le débat au sein de la communauté djihadiste saoudienne sur la question de savoir s'il fallait combattre en Irak ou en Arabie Saoudite a été tranché à l'été 2004 en faveur de l'Irak. La capture de Saddam Hussein en décembre 2004 a contribué au renforcement du recrutement pour l'Irak. En effet, cela a apparemment résorbé la peur initiale au sein des communautés islamistes, selon laquelle le combat en Irak pourrait bénéficier au parti Baath. Le premier siège de Falloudjah en avril 2004 a lui aussi contribué à galvaniser le soutien, parce qu'il était perçu par les djihadistes comme une victoire ayant conduit à la création d'un bastion du groupe de Zarqaoui à Falloudjah. La mise à jour d'événements comme le scandale d'Abou Ghraïb et la campagne de prises d'otages de 2004 ont aussi renforcé le recrutement pour l'Irak. Depuis la fin de 2004, le nombre de Saoudiens entrant en Irak semble avoir décru, en partie du fait du renforcement du contrôle aux frontières et du démantèlement des réseaux de recrutement ${ }^{16}$. Cependant, il y a encore un flux conséquent de Saoudiens et d'autres militants étrangers vers l'Irak ${ }^{17}$.

Le nombre de Saoudiens partis en Irak est contesté et ne sera probablement jamais connu. Le ministère de l'Intérieur saoudien n'a, jusqu'à présent, pas produit de chiffres officiels. Les estimations les plus basses - environ 350 en août 2005 - proviennent de sources proches du gouvernement ${ }^{18}$. Les estimations les plus élevées viennent de figures de l'opposition saoudienne comme Muhammad al-Mas'ari, qui a affirmé que, dès le mois d'octobre 2005, il y a eu 5000 Saoudiens à Bagdad ${ }^{19}$. Le chiffre exact se trouve probablement quelque part entre les deux. Vers le milieu de l'année 2005, des agents de sécurité officiels auraient avancé un chiffre situé entre 2000 et $3000{ }^{20}$. En répertoriant les Saoudiens arrêtés ou morts, nous en sommes arrivés à un total de 421 individus ( 248 « martyrs » et détenus nommés et 173 détenus anonymes). Cette liste est sans aucun doute incomplète; certains individus continuent probablement à se battre en Irak, d'autres sont peut-être morts sans être mentionnés dans la littérature djihadiste et des indices d'ordre anecdotique laissent croire qu'un certain nombre d'individus se seraient rendus en Irak puis seraient retournés discrètement en Arabie Saoudite ${ }^{21}$. Cependant, rien ne permet de laisser croire que le nombre dépasserait les 1 500. Cela est d'autant plus vrai que le chiffre total de combattants étrangers en Irak ne semble pas avoir dépassé 3000 , quel que soit le moment considéré ${ }^{22}$.

Selon une perception dominante, les Saoudiens seraient largement surreprésentés parmi les combattants étrangers en Irak ${ }^{23}$. Les estimations s'échelonnent entre $12 \%$ et $61 \%{ }^{24}$. Le décalage considérable entre les différentes estimations atteste de la rareté des informations crédibles sur l'insurrection, ainsi que de la nature très controversée du débat sur le rôle des Saoudiens en Irak. L'étude en détail du « contingent » saoudien, notamment à partir des sources ouvertes disponibles, n'en est que plus importante.

Saoudiens "martyrs " de notre échantillon étaient de jeunes hommes qui avaient en moyenne 23 ans en 2003 (le plus jeune avait 18 ans, le plus vieux, 39 ans). Cette moyenne relativement basse semble indiquer que les Saoudiens en Irak représentent une nouvelle génération de moudjahiddin ${ }^{25}$. En effet, seuls 9 des 205 Saoudiens " martyrs » sont connus pour avoir eu des expériences de combat préalables en Afghanistan, en Bosnie ou ailleurs. Cela montre que le recrutement pour l'Irak est un phénomène distinct et spontané et que la relation à l'ancien réseau al-Qaïda reste marginale.

Bien que les informations disponibles sur l'origine socio-économique des combattants soient éparses, elles semblent indiquer qu'il s'agit d'un groupe très diversifié. Certains 
viennent de milieux très pauvres, d'autres, de familles riches et privilégiées. Les informations sur leur niveau d'éducation sont également rares, mais indiquent que beaucoup avaient une éducation supérieure : 14 combattants sur les 16 pour lesquels nous disposons d'informations sur le niveau d'éducation ont bénéficié d'une éducation supérieure et l'un d'entre eux avait un doctorat. Nous connaissons le métier de 26 individus: 14 étudiants, 3 employés du secteur privé, 2 appartenant au clergé gouvernemental, 2 infirmiers, 2 officiers de police / militaires, un enseignant, un mécanicien et un météorologue.

L'échantillon est également diversifié au niveau de l'origine géographique et tribale. Les informations dont nous disposons sur ces origines montrent que toutes les régions (à l'exception du Jizan dans l'extrême Sud) sont représentées (voir tableau 1) ${ }^{26}$. Rapportées à la distribution générale de la population saoudienne, elles montrent que les régions les plus surreprésentées sont celles, centrales, de Riyad et Qasim, ainsi que les régions du Nord-Ouest de Tabuk, Jawf et Ha'il ${ }^{27}$. Les régions les plus sousreprésentées sont les régions du Sud de Jizan, Najran, Asir et Baha. Cela contredit fermement la perception dominante (dérivée des profils des preneurs d'otage du 11 septembre 2001) des régions du Sud de l'Arabie Saoudite comme terreau d'exportation principal de militants saoudiens ${ }^{28}$.

21 La présence notable d'habitants du Nord suggère que la proximité géographique au champ de bataille a influencé le recrutement pour l'Irak. Les habitants du Nord ont, semble-t-il, eu un accès privilégié à l'Irak, leur permettant de s'infiltrer plus facilement. Il est aussi possible qu'ils aient été plus sensibles à la présence américaine qui était davantage visible dans le Nord au début $2003{ }^{29}$. Les loyautés tribales ont aussi pu jouer un rôle, puisqu'un certain nombre de tribus sont transversales à la frontière. Les «tribus partagées» comme les Shammar sont ainsi largement présentes dans notre échantillon (voir tableau 2). Il peut être intéressant de noter que la distribution tribale de notre échantillon est très large et couvre la plupart des grandes tribus. En excluant celles du Nord, il est donc difficile d'affirmer qu'il y aurait un facteur tribal dans le recrutement de Saoudiens pour l'Irak.

Tableau 1 : origine géographique (par région) des militants saoudiens

\begin{tabular}{|l|l|l|l|}
\hline & Saoudiens en Irak & Population globale & Membres de QAP \\
\hline \hline Riyadh & $36 \%$ & $21 \%$ & $53 \%$ \\
\hline \hline Mecca & $19 \%$ & $23 \%$ & $18 \%$ \\
\hline \hline Qasim & $13 \%$ & $5 \%$ & $7 \%$ \\
\hline \hline Jawf & $9 \%$ & $2 \%$ & $2 \%$ \\
\hline \hline Eastern & $7 \%$ & $15 \%$ & $5 \%$ \\
\hline \hline Ha'il & $6 \%$ & $3 \%$ & $0 \%$ \\
\hline \hline Tabuk & $5 \%$ & $3 \%$ & \\
\hline
\end{tabular}




\begin{tabular}{|l||l|l|l|}
\hline Baha & $2 \%$ & $3 \%$ & $0 \%$ \\
\hline \hline Medina & $1 \%$ & $7 \%$ & $4 \%$ \\
\hline \hline Nord & $1 \%$ & $1 \%$ & $0 \%$ \\
\hline \hline Asir & $1 \%$ & $9 \%$ & $7 \%$ \\
\hline \hline Jizan & $0 \%$ & $6 \%$ & $2 \%$ \\
\hline \hline Najran & $0 \%$ & $2 \%$ & $0 \%$ \\
\hline
\end{tabular}

Tableau 2 : origine tribale des militants saoudiens

\begin{tabular}{|c|c|c|c|}
\hline \multicolumn{2}{|c|}{ Saoudiens en Irak } & \multicolumn{2}{|c|}{ Membres de QAP } \\
\hline Inconnu & $50,2 \%$ & Inconnu & $63,8 \%$ \\
\hline Utayba & $7,8 \%$ & Harb & $7,5 \%$ \\
\hline Shammar & $6,8 \%$ & Ghamid & $4,8 \%$ \\
\hline Qahtan & $6,3 \%$ & Utayba & $3,5 \%$ \\
\hline Ghamid & $4,9 \%$ & Shammar & $2,6 \%$ \\
\hline Mutayr & $4,4 \%$ & Subay & $2,2 \%$ \\
\hline Harb & $3,4 \%$ & Mutayr & $2,2 \%$ \\
\hline Banu Tamim & $2,4 \%$ & Qahtan & $2,2 \%$ \\
\hline Banu Shihr & $2,4 \%$ & Banu Khalid & $2,2 \%$ \\
\hline Banu Khalid & $1,5 \%$ & Anaza & $1,7 \%$ \\
\hline Anaza & $1,5 \%$ & Shihr & $1,7 \%$ \\
\hline Dawasir & $1,5 \%$ & Dawasir & $1,7 \%$ \\
\hline Banu Harith & $1,5 \%$ & Suhul & $1,7 \%$ \\
\hline Subay & $1,0 \%$ & Umran & $1,3 \%$ \\
\hline Zahran & $1,0 \%$ & Quraysh & $0,9 \%$ \\
\hline Suhul & $1,0 \%$ & Fadhl & $0,9 \%$ \\
\hline
\end{tabular}




\begin{tabular}{|l|l|l|l|}
\hline Sulami & 1,0 \% & Zahran & O,4 \% \\
\hline \hline Sharif & $0,5 \%$ & \\
\hline \hline Quraysh & $0,5 \%$ & \\
\hline \hline Banu Malik & $0,5 \%$ & \\
\hline
\end{tabular}

24 La diversité socio-économique, géographique et tribale nous dit deux choses importantes. Premièrement, elle montre que la militance n'est pas limitée à un secteur particulier de la société saoudienne (les "pauvres», les «habitants du Sud», les "Bédouins", etc.). Deuxièmement, elle montre que la sympathie pour la cause irakienne traverse toute la société saoudienne. L'importance de ce point devient plus claire lorsque l'on compare les Saoudiens en Irak à un échantillon de 240 militants «domestiques» du groupe "al-Qaïda sur la Péninsule arabique » (QPA). Les Saoudiens en Irak représentent un groupe plus diversifié que QPA, que ce soit en termes socioéconomiques, géographiques ou tribaux (voir tableau 1 et $2{ }^{30}{ }^{30}$. C'est probablement le résultat des perceptions différenciées de la légitimité du combat en Irak et à l'intérieur de l'Arabie Saoudite ${ }^{31}$. Il était bien plus facile d'inciter les gens à combattre les militaires américains en Irak que de faire exploser des voitures dans les rues de Riyad.

Les Saoudiens en Irak semblent avoir été motivés par des facteurs diversifiés. Les motivations déclarées étaient de deux types. Le premier, altruiste: libérer les coreligionnaires musulmans de l'occupation américaine. Le second, plus personnel: devenir un martyr et aller au paradis. Dans les biographies sur les sites Internet djihadistes, ces motivations sont répétées par des phrases clichés, au point que l'on peut douter de leur sincérité ${ }^{32}$.

Cependant, au vu de sources additionnelles comme des entretiens avec des militants et recruteurs, il semble que la plupart des recrues saoudiennes aient eu une foi véritable et un désir de devenir des martyrs, tout du moins au moment où ils sont entrés en Irak ${ }^{33}$. Pour certains, la perspective d'aller au paradis semblerait avoir été plus importante que celle d'atteindre des buts politiques ${ }^{34}$. Cette obsession du martyre peut, en partie, avoir été motivée par des dynamiques de groupe, et partiellement entretenue par les recruteurs pour des raisons opérationnelles et tactiques.

Il est difficile de savoir si les recrues saoudiennes ont été plus préoccupées par le martyre que les volontaires d'autres pays. Beaucoup de commentateurs ont suggéré que tel était le cas, en se référant aux affirmations américaines ou irakiennes selon lesquelles les Saoudiens seraient surreprésentés parmi les auteurs d'attentats-suicides. Certaines sources djihadistes évoquent l'obsession des Saoudiens pour le martyre, mais les preuves fournies restent toujours d'ordre anecdotique ${ }^{35}$. Peut-être le seul argument raisonnablement convaincant est-il le ratio des Saoudiens tombés « en martyrs » et les Saoudiens capturés en Irak. Il y a un décalage important entre la proportion des Saoudiens sur la liste des martyrs arabes en Irak d'une part (à peu près $60 \%$ ), et la proportion des Saoudiens parmi les citoyens étrangers détenus par les Etats-Unis en Irak de l'autre (à peu près $10 \%$ ) ${ }^{36}$. Même en tenant compte des biais possibles de ces listes, cela semblerait suggérer que le taux de décès serait plus élevé pour les Saoudiens que pour d'autres groupes ${ }^{37}$. Plusieurs raisons peuvent être avancées pour expliquer 
cela. Les Saoudiens peuvent avoir été utilisés comme "chair à canon " par les chefs de l'insurrection irakienne, ils peuvent avoir été des combattants moins expérimentés ou ils peuvent avoir été plus motivés par des opérations de guerre à haut risque et/ou suicidaires.

Il est important de souligner que même s'il était possible de prouver que le désir de mourir en martyr était particulièrement prégnant parmi les djihadistes saoudiens, cela ne signifierait pas que leur engagement ne serait pas également politique. Il est parfaitement compatible d'être motivé à la fois par une frustration politique et une religiosité extrême. En réalité, les deux sont liés : si une lutte n'est pas considérée comme un djihad légitime, la mort en martyr n'est pas possible.

Des facteurs politiques sont en effet fréquemment cités parmi les motivations pour se rendre en Irak. Au-delà de l'invasion elle-même, des événements spécifiques comme le scandale d'Abou Ghraïb et les sièges successifs de Falloudjah sont cités par plusieurs recrues comme des éléments importants dans leur décision de partir pour le champ de bataille irakien ${ }^{38}$. Non moins important est l'effet de ces événements sur les parents. Beaucoup de recrues potentielles ne seraient pas allées en Irak sans la permission de leurs parents. Certains, qui avaient initialement refusé, ont changé d'avis après avoir été indignés par les images provenant de l'Irak ${ }^{39}$.

30 Cependant, pour peu que l'on veuille bien lire entre les lignes des motivations affichées, il semblerait que des facteurs plus largement sociaux puissent également avoir joué un rôle non négligeable. Un certain nombre de recrues étaient clairement inspirées par des amis et des membres de la famille partis à l'étranger sous la bannière du djihad avant eux ${ }^{40}$. Certains semblent avoir voulu suivre leurs pères et leurs oncles, d'autres ont cru qu'ils allaient rencontrer au paradis des amis tombés en martyrs s'ils tombaient en martyrs à leur tour. D'autres encore semblent avoir senti une pression sociale de la part de leurs proches. La nouvelle du martyre d'un jeune Saoudien se propage en effet rapidement au sein de sa famille et de son voisinage, et ce, d'autant plus que les familles tiennent souvent dans leur maison des cérémonies de condoléances largement affichées. Dans certains villages ou quartiers, comme le district de Sawaydi à Riyad ou la zone de Thaqba près de Dammam, la fréquence de ces nouvelles implique que la plupart des résidents connaissent quelqu'un qui est parti pour l'Irak ${ }^{41}$. Cela a, en retour, abaissé leur seuil d'engagement dans cette forme d'activisme, un phénomène bien connu des "jeux d'assurance» dans la théorie du choix rationnel. Ces réseaux sociaux étaient aussi importants pour les modes de recrutement des Saoudiens en partance pour l'Irak.

31 Les Saoudiens ont été mobilisés pour le combat en Irak de différentes manières. Certains étaient enrôlés par un recrutement classique et hiérarchique. D’autres ont subi un processus « par le bas " par une radicalisation solitaire ou au sein d'un cercle d'amis. Mais ce n'est que dans un deuxième temps que cette forme de radicalisation a conduit, de la propre initiative de l'individu ou du groupe en question, à la recherche de «facilitateurs ».

Les recrutements hiérarchiques se sont produits en divers endroits et de diverses manières. Beaucoup de ces types de recrutement ont suivi la logique de liens sociaux préexistants et ont eu lieu dans le cadre de rassemblements privés. La Mecque a été une autre arène majeure pour des recruteurs ou des incitateurs, car des milliers de jeunes Saoudiens y vont pendant le hajj ou les derniers dix jours du Ramadan. La Mecque offre en effet d'excellentes opportunités au recrutement, les pèlerins étant plus ouverts à de 
nouvelles rencontres et expériences religieuses. De tels rassemblements rendent, en outre, la surveillance policière moins aisée ${ }^{42}$. Pour beaucoup de jeunes Saoudiens, le fait de partir à la Mecque est aussi une manière d'échapper au contrôle parental. Ainsi, nombre de Saoudiens ont décidé de partir en Irak après avoir passé un certain temps à la Mecque ${ }^{43}$.

L'incitation par des chefs religieux constitue un autre mode plus indirect de recrutement hiérarchique. Des faisceaux d'indices tendent à confirmer que des imams locaux et des clercs importants ont encouragé la participation à la résistance irakienne 44. Certains des Saoudiens de notre échantillon ont explicitement affirmé avoir été inspirés par des prêches entendus dans une mosquée locale ${ }^{45}$. Dans une biographie, il est dit que Abdallah bin Jibrin, l'un des docteurs en religion les plus connus du royaume saoudien, s'est rendu à la maison de la famille pour congratuler cette dernière pour le martyre du fils ${ }^{46}$.

Il y aussi beaucoup d'exemples de recrutement " par le bas " pour l'Irak. Internet a sans doute joué un rôle majeur dans «l'auto-radicalisation" de nombre de jeunes Saoudiens. Depuis le début de la guerre en Irak, les sites Internet djihadistes ont été submergés par des images, des enregistrements vidéo et de la propagande écrite sur la souffrance du peuple irakien sous l'occupation américaine ${ }^{47}$. Internet a aussi facilité la communication entre des recrues potentielles et ceux que l'on appelle les "gatekeepers» (littéralement: les "portiers»), ceux qui donnent des conseils pratiques facilitant l'accès à l'Irak. Des instructions écrites renseignant sur la manière de rejoindre le djihad Irakien ont aussi circulé sur Internet ${ }^{48}$. Cependant, il semblerait que la plupart des recrutements par le bas aient suivi des liens sociaux informels. Dans beaucoup de communautés islamistes, les gatekeepers sont bien connus, les recrues potentielles savent donc à qui s'adresser ${ }^{49}$.

Dans une majorité de cas, le processus de recrutement "par le bas» a été une expérience collective, des pairs ou des groupes d'amis décidant de partir pour l'Irak ensemble : la plupart des personnes de notre échantillon sont allées en Irak avec au moins un ami proche, frère ou cousin ${ }^{50}$.

Beaucoup de biographies de martyrs saoudiens en Irak mentionnent la date d'arrivée en Irak. Beaucoup sont partis pendant ou autour du mois du Ramadan, particulièrement en 2004. La raison principale peut en être " historique »: la seconde bataille de Falloudjah a eu lieu lors du Ramadan de l'an 1425, soit en novembre 2004. Beaucoup de biographies accordent une grande importance au fait qu'un individu soit parti pour l'Irak au moment du Ramadan. Si une personne est tombée en martyr pendant les derniers dix jours du Ramadan ou (encore mieux) au moment du Laylat alQadr (27e jour du Ramadan), l'anniversaire de la première révélation coranique, cela est aussi généralement souligné ${ }^{51}$. Il semblerait que la croyance à la valeur du martyre pendant le Ramadan ait affecté les modes de recrutement de Saoudiens pour l'Irak ${ }^{52}$.

Les Saoudiens ont emprunté des itinéraires divers pour se rendre en Irak. Les routes préférées ont varié avec le moment et souvent en rapport à la mise en place de contremesures aux différents points de transit des frontières irakiennes. Ceux qui sont partis au Kurdistan juste avant la guerre ont transité par le Qatar et l'Iran. Jusqu'au milieu de 2004 environ, la frontière irako-saoudienne était assez poreuse pour être traversée sans difficulté. Il s'agissait de l'itinéraire préféré de ceux qui n'avaient pas de documents de voyage (beaucoup d'extrémistes connus ont vu leur passeport confisqué par la police). Depuis cette période, les autorités saoudiennes ont renforcé le contrôle aux frontières 
et, par conséquent, la majorité des recrues est partie au Yémen, puis en Syrie et en Irak. Les forces américaines ont eu des difficultés à contrôler la frontière irako-syrienne au Nord de l'Euphrate, car, comme il y avait peu de ponts assez solides pour supporter des véhicules blindés, la plupart des unités est restée sur la rive sud ${ }^{53}$. Un an plus tard, les forces américaines ont conduit des opérations massives pour contrôler cette région, ce qui a réduit le flux des militants étrangers arrivant par la frontière syro-irakienne. Il y a également eu des cas de Saoudiens entrant par la Jordanie et la Turquie.

Une fois en Irak, la plupart des volontaires saoudiens sont allés là où leur «facilitateur » leur a conseillé d'aller ${ }^{54}$. La majorité des Saoudiens en Irak ont rejoint le réseau de Al-Zarqaoui, de loin le plus internationalisé des groupes militants en Irak ${ }^{55}$. Les biographies évoquent des communications fréquentes par des lignes téléphoniques ouvertes entre l'Arabie Saoudite et l'Irak. Certains volontaires ont régulièrement appelé leurs familles depuis l'Irak, d'autres n'ont plus jamais donné de nouvelles. Cependant, si une personne est tuée, un de ses compagnons appelle généralement la famille du martyr pour l'en informer et pour la féliciter. La nouvelle de la mort d'un martyr saoudien en Irak est souvent largement diffusée et célébrée dans son quartier d'origine ainsi que dans celui de sa famille. Le numéro de téléphone portable du père ou du frère du martyr - accompagné de l'adresse et des indications relatives à la maison de la famille - est alors publié sur Internet pour que n'importe qui puisse lui rendre visite, l'appeler pour la féliciter ou lui présenter ses condoléances.

Le rôle précis du contingent saoudien dans l'insurrection irakienne est très difficile à déterminer parce que l'on en sait très peu de la composition et du fonctionnement interne de l'insurrection en général. Dans les premières phases de l'insurrection, le rôle des étrangers semble avoir été exagéré. Dans la phase actuelle, les Saoudiens seraient "numériquement faibles mais opérationnellement importants ", description qui verse parfois dans le cliché rhétorique. Les étrangers, qui représenteraient entre $5 \%$ et $10 \%$ du nombre total d'insurgés sunnites, auraient, dit-on, introduit des tactiques nouvelles et brutales, initié la violence sectaire et seraient les auteurs de la plupart des attentatssuicides en Irak ${ }^{56}$.

Il semblerait que les Saoudiens aient essentiellement opéré dans les rangs hiérarchiquement inférieurs des organisations militantes en Irak ${ }^{57}$. Les volontaires saoudiens ont mis en œuvre un nombre important d'attaques-suicides, y compris certaines des plus spectaculaires de l'histoire de l'insurrection armée ${ }^{58}$. Au moins 33 personnes de notre échantillon de 205 martyrs sont mortes dans des attentats-suicides. Nombre d'indices suggèrent que des groupes djihadistes en Irak ont modelé le recrutement et l'affectation des Saoudiens pour l'exécution d'attentats-suicides. Début 2005, un Saoudien, Ahmad al-Shayea, a survécu de justesse à un tel attentat à Bagdad. Il a, par la suite, affirmé aux médias que l'opération avait été effectuée contre son gré on lui a ordonné de conduire une camionnette sans savoir qu'elle était chargée d'explosifs ${ }^{59}$. Les médias officiels saoudiens se sont saisis de l'histoire pour convaincre le public du fait que les recrues saoudiennes étaient généralement dupées et exploitées par des groupes irakiens qui ne voulaient des Saoudiens que pour financer et mettre en pratique de telles opérations-suicides ${ }^{60}$. L'instrumentalisation probable des Saoudiens dans le cadre d'attentats-suicides montre surtout que ces opérations doivent être replacées et analysées dans un contexte organisationnel plus large et qu'elles ne peuvent pas uniquement être envisagées comme choix individuel. 

quelque peu surestimées par les commentateurs. Cela pourrait être lié à la tendance plus générale à exagérer l'importance de la composante externe de l'insurrection irakienne afin de minimiser son soutien local en Irak. Même si les Saoudiens ont contribué à bon nombre d'attentats-suicides, il semblerait que ceux-ci n'aient eu qu'un impact limité sur la stratégie globale adoptée par les groupes insurgés en Irak ces dernières années. Qui plus est, le nombre de Saoudiens partant pour l'Irak a tendanciellement diminué depuis le début 2005, et la mort d'al-Zarqaoui en juin 2006 ne semble pas contribuer à faciliter l'entrée en Irak.

Beaucoup ont mis en garde contre la menace à la sécurité intérieure saoudienne que représenterait le retour de combattants d'Irak ${ }^{61}$. Dans le passé, des vétérans de l'Afghanistan ont joué un rôle important dans la violence à l'intérieur de l'Arabie Saoudite. Il n'est pas exclu qu'il en soit de même pour des « vétérans d'Irak » brutalisés. Alors même que ce risque ne doit pas être sous-estimé, il faut cependant garder à l'esprit que des différences majeures distinguent les «Arabes afghans» des «Arabes irakiens ». Parmi celles-ci, concernant la taille du «contingent», il faut noter que le nombre de Saoudiens en Irak ne dépasserait pas 1500 et que beaucoup d'entre eux meurent sur le champ de bataille. Le mode de rapatriement est, lui aussi, différent. Contrairement aux retours d'Afghanistan du début 2002, les vétérans d'Irak ne rentreront que sporadiquement et au fur et à mesure. Le problème qu'ils sont susceptibles de constituer pourrait ainsi être abordé plus efficacement. à la sécurité, les anciens d'Irak seront suivis de très près par les forces de sécurité. approche diversifiée et mesurée qui a montré certains résultats. Cependant, sur le long terme, la question principale est de savoir si le royaume sera capable et désireux de contrer les formes les plus radicales du nationalisme pan-islamique qui motivent le recrutement vers les zones de djihad à l'étranger et inspirent les attaques contre des cibles occidentales à l'intérieur de l'Arabie Saoudite. Ce n'est pas tâche facile, car les prêcheurs populistes sont pléthore dans le royaume et le nationalisme pan-islamique est en progrès au niveau international. De plus, les Etats-Unis continuent de raviver le feu pan-islamique avec leur politique étrangère, comme l'ont montré les événements récents au Liban. De ce point de vue, les combattants saoudiens en Irak ne seront probablement pas la dernière génération de volontaires en partance pour l'étranger.

\section{NOTES}

1.. Traduction de l'anglais par Christian Olsson.

2.. Parmi les exceptions, on trouve Obaid N., Cordesman, A., « Saudi militants in Iraq: assessment and kingdom's response », Washington, Center for Strategic and International Studies, 2005 ; Paz R., « Arab volunteers killed in Iraq: an analysis », PRISM Papers $3, \mathrm{n}^{\circ} 1,2005$.

Cultures \& Conflits, 64 | hiver 2006 
3.. Des problèmes de fiabilité des sources se posent certainement quant aux biographies des martyrs publiées sur Internet. Les auteurs sont le plus souvent anonymes et les informations ne peuvent pas toujours être vérifiées. Les propagandistes djihadistes ont beaucoup de raisons de vouloir embellir les histoires qu'ils rapportent et gonfler le nombre de martyrs. Cependant, cela ne justifie pas le rejet total de ces biographies comme de purs artefacts (Obaid N., Cordesman A., op. cit., p. 22). Beaucoup de décès proclamés sur Internet ont été corroborés par des rapports parus dans la presse saoudienne et fondés sur des témoignages de membres de la famille de la personne décédée. Nombre de biographies contiennent également les numéros de téléphone et adresses des membres de la famille du martyr. Les martyrs saoudiens sont surreprésentés dans ces listes parce que celles-ci sont constituées justement par des Saoudiens. Par conséquent, elles ne nous disent pas grand-chose sur la taille relative des différents « contingents » étrangers. En revanche, si l'on extrait les biographies des Saoudiens et si on les considère en tant que groupe particulier, il n'y pas de raison pour que le nombre obtenu ne soit pas raisonnablement représentatif du nombre de Saoudiens partis en Irak.

4.. NDT : Dans le premier cas, il s'agit de l'Afghanistan après l'invasion soviétique de 1979, la seconde fois, de l'Afghanistan après l'invasion américaine de 2001.

5.. Par exemple, le dirigeant d'Al-Qaïda, Khalid Shaykh Muhammad, aurait affirmé à des interrogateurs que, dans n'importe quel camp en Afghanistan en 2001, 70 \% des recrues seraient des Saoudiens ; voir : The 9/11 Commission Report: Final Report of the National Commission on Terrorist Attacks Upon the United States, New York, W.W. Norton \& Co., 2004.

6.. Saleh Ambah F., « Iraq: spinning off Arab terrorists? », Washington Post, 8 février 2005.

7.. Windrem R., « Saudi Arabia's ambitious al-Qaida fighter », NBC News, 11 juillet 2005. 8.. Abd al-Muhsin al-Ubaykan, « ma yajri fi'l-'iraq laysa jihadan [Ce qui se passe en Irak n'est pas le djihad] », al-Sharq al-Awsat, 4 novembre 2004.

9.. « jam'min al-'ulama al-sa'udiyyin yuwajjihuna khatiban maftuhan li'l-sha'b al-'iraqi » [Lettre ouverte adressée directement au peuple irakien par un groupe de docteurs saoudiens], www.islamtoday.net, 5 novembre 2004.

10.. Voir la déclaration d'Ayidh al-Qarni's sur al-Jazeera le 14 novembre 2004, l'article de Salman al-Awda's dans le journal al-Riyadh ( ${ }^{\circ}$ du 20 novembre 2004) et l'entretien de Safar al-Hawali's avec al-Ukaz le 19 mai 2005.

11.. Des universitaires liés au gouvernement, comme Salih al-Luhaydan, ont exprimé des points de vue similaires ; voir Myers L., " More evidence of Saudi doubletalk », Newsweek, 26 avril 2005.

12.. Hegghammer T., « Global jihadism after the Iraq war », Middle East Journal 60, n¹, 2006.

13.. Lia B., «The ansar al-islam group revisited », Studies in Conflict and Terrorism 30, $n^{\circ} 3,2007$ [à paraître].

14.. MacFarquhar N., «For Arabs, new jihad is in Iraq », New York Times, 2 avril 2003 ; Bazzi M., " A new jihad vs America? Iraq fight draws Arab men », Newsday, 20 juillet 2003 ; Huband M., « Saudis in Iraq "preparing for a holy war" ", Financial Times, 18 août 2003 ; Waterman S., « US holds Saudi, Arab fighters in Iraq », United Press International, 27 août 2003 ; Saleh Ambah F., « Saudi fighters cross border to kill infidels », Washington Times, 31 août 2003 ; « Four Saudi militants held near border 
with Iraq: police », Agence France presse, 13 octobre 2003 ; Schwartz S., « An unwelcome Saudi export », Weekly Standard, 2 février 2004.

15.. Gedye R., « Iraq becomes a battleground in war on infidels », Daily Telegraph, 19 août 2003.

16.. Smith C.S., « U.S. contends campaign has cut suicide attacks », New York Times, 5 août 2005.

17.. En octobre 2006, le forces armées américaines ont affirmé qu'» entre 50 et 60 combattants étrangers s'infiltrent en Irak chaque mois » et que « la plupart viennent d'Arabie Saoudite, du Soudan, d'Egypte ou de Syrie » ; Grier P., « Iraq war draws foreign jihadists, but not in droves ", Christian Science Monitor, 3 octobre 2006.

18.. Obaid N., Cordesman A., op. cit.

19.. Aziz S., " Saudi fighters "join resistance" in Iraq ", al-Jazeera English Edition, 28 octobre 2003. Saad al-Faqih a affirmé en août 2003 qu'il y avait 3000 Saoudiens en Irak; « Bremer urges Syria to stop fighters entering Iraq », Reuters, 19 août 2003.

20.. al-Sarhan S., « al-qa'ida fi'l-sa'udiyya: min al-tashkik ila al-ghazal [Al-Qaïda en Arabie Saoudite : de la suspicion au flirt] », al-Sharq al-Awsat, 20 mai 2005 ; al-Dhaidi M., « al-irhabiyyun fi'l-'iraq yuridun al-sa'udi imma intihariyyan aw mumawwilan... faqat [Les terroristes en Irak ne veulent des Saoudiens qu'en tant qu'auteurs d'attentats-suicides ou en tant que source de financement] ", al-Sharq al-Awsat, 20 septembre 2005.

21.. Ambah, «Saudi fighters cross border to kill infidels »; entretien de l'auteur avec Saud al-Sarhan et Yusuf al-Dayni, 2005.

22.. En octobre 2005, le ministre irakien de l'Intérieur a affirmé que les militants arabes étrangers étaient maintenant moins de 1000 « en comparaison de 2500 et 3000 il y a six mois "; al-Khalidi S., " Iraq says Zarqawi sending some militants back home ", Reuters, 2 octobre 2005. La même estimation a été donnée par les sources officielles américaines à la fin 2005 ; Eisenstadt M., White J., « Assessing Iraq's Arab insurgency ", Policy Focus, Washington DC, Washington Institute for Near East Policy, 2005. En octobre 2005, les forces armées américaines ont affirmé que seulement 312 des 10000 détenus insurgés étaient des étrangers ; Filkins D., « Foreign fighters captured in Iraq come from 27, mostly Arab, lands ", New York Times, 21 octobre 2005.

23.. Glasser S.B., " "Martyrs" in Iraq mostly Saudis », Washington Post, 15 mai 2005 ; Bloom M., « Grim Saudi export - suicide bombers », Los Angeles Times, 17 juillet 2005. 24.. Obaid N., Cordesman A., op. cit.; Paz R., « Arab volunteers killed in Iraq: an analysis ». Les deux estimations sont très problématiques. L'étude du CSIS ne fait référence qu'à des « sources des renseignements " pour justifier ses chiffres étrangement précis sur la composition de l'insurrection. L'étude de Paz est fondée sur une ancienne version du document Internet djihadiste, Martyres arabes en Mésopotamie, qui, dans la mesure où il a été essentiellement compilé par des Saoudiens, est susceptible d'avoir prêté une attention particulière aux martyrs saoudiens.

25.. Cette moyenne est de quatre années inférieure à la moyenne de l'âge des militants internes au pays, connus sous le nom d'Al-Qaïda sur la péninsule arabique, la majorité desquels sont des vétérans de l'Afghanistan ; Hegghammer T., « Terrorist recruitment and radicalisation in Saudi Arabia », Middle East Policy 13, n% 2006.

26.. Nous connaissons l'origine géographique de 151 des 205 militants. 
27. Les chiffres globaux sur la population viennent du Département central des statistiques, « Demographic Survey Report 1421H-2001 », ministère saoudien de la Planification, http://www.planning.gov.sa.

28. Ces preuves s'ajoutent à celles de notre étude sur les milieux d'origine de 240 militants saoudiens à l'intérieur du royaume, qui montre aussi que les régions du Sud ne sont pas surreprésentées; Hegghammer T., « Terrorist recruitment and radicalisation in Saudi Arabia », op. cit.

29.. L'armée de l'air américaine a utilisé des installations dans le nord de l'Arabie Saoudite lors de la guerre contre l'Irak ; Bradley J.R., " Saudi town sows seeds of revolution ", Washington Times, 26 janvier 2004

30.. Hegghammer T., " Terrorist recruitment and radicalisation in Saudi Arabia », op. cit.

31.. MacFarquhar N., " Saudis support Jihad in Iraq, not back home », New York Times, 23 avril 2004.

32.. al-Jihad M., " "shuhada" ard al-rafidayn [Martyrs du pays des deux rivières] », 2005.

33.. Abdul-Ahad G., " In hideout, foreign arabs share vision of "martyrdom" ", Washington Post, 9 novembre 2004.

34.. La biographie de Faris al-Badiawi est un exemple parlant. Celui-ci aurait, dit-on, commencé à pleurer après avoir survécu à sa première bataille. Il a raconté à ses camarades « Si je ne tombe pas en martyr dans la prochaine bataille, je rentrerai chez ma famille » ; voir al-Jihad M., op. cit. Toutes les biographies citées dans cet article viennent de cette source lorsque leurs références ne sont pas explicitement spécifiées. 35.. « Bin Laden's former mufti Saudi cleric musa Al-Qarni: Saudi youth are exceptional in terms of sacrifice in their love of Jihad and their desire to reach paradise; fighting the American aggressors in Iraq is Jihad and constitutes a legitimate and obligatory defense », MEMRI Special Dispatch, $\mathrm{n}^{\circ}$ 1124, 2006.

36. Paz R., dans « Arab volunteers killed in Iraq: an analysis », dit que 94 martyrs sur une liste en comprenant 154 auraient été Saoudiens. En octobre 2005, les militaires américains ont affirmé que 32 de leurs détenus étrangers en Irak auraient été Saoudiens ; Filkins D., « Foreign fighters captured in Iraq come from 27, mostly Arab, lands ", New York Times, 21 octobre 2006.

37.. Les Egyptiens, par exemple, étaient absents de l'échantillon de Paz mais constituaient le plus grand « contingent » $(25 \%)$ parmi les détenus.

38. Voir par exemple la biographie d'Abd al-Aziz al-Rashid.

39. Voir par exemple la biographie de Bandar al-Badri.

40.. Par exemple, Nasir al-Qahtani et Muhammad al-Qahtani, respectivement le frère et fils de Ayidh al-Qahtani, mort au Tadjikistan en 1995, sont tous deux allés en Irak.

Ahmad al- Dhahiri avait un père qui avait combattu durant la première guerre d'Afghanistan. Asim al-Dhahiri est allé en Irak après que son frère Ahmad Fawaz alZahrani soit allé en Irak immédiatement après la mort de son ami Salwah al-Fahmi à Bagdad.

41. De la même façon, certaines villes et communautés aux Etats-Unis fournissent un nombre disproportionné de recrues aux militaires américains en Irak ; Jonsson P., « In small towns, military values a big draw », Christian Science Monitor, 19 octobre 2005. 42.. En avril 2005, la police saoudienne a démantelé un réseau de recruteurs à la Mecque ; " masadir amniyya : khaliyat makka kanat mutakhassisa bi-irsal muqatilin li'lzarqawi ‘abr al-yaman wa suria [La cellule de la Mecque spécialisée dans l'envoi de 
combattants à al-Zarqaoui au travers du Yémen et de la Syrie] », al-Sharq al-Awsat, 23 avril 2005.

43.. Voir par exemple les biographies de Majid al-Mutayri et Fahd al-Shihri.

44.. Faramarzi S., « Saudi clerics point militants toward Iraq », Associated Press, 24

janvier 2005.

45.. Voir par exemple la biographie de Mahir al-Juhani.

46.. Voir par exemple la biographie de Faris al-Harbi.

47.. Hegghammer T., « Global jihadism after the Iraq war », op. cit.

48.. al-Duktur al-Islami, « hadhihi hiya al-tariq ila al-'iraq [Voici le chemin vers

l'Irak] », Muntada al-Hikma, 2005.

49.. Entretien de l'auteur avec plusieurs personnes en Arabie Saoudite restées anonymes, novembre 2005. Voir aussi Abu-Nasr D.,» Underground network helps Saudis go to Iraq for Jihad », Associated Press, 24 février 2005.

50.. Voir par exemple la biographie de Ziyad al-Thunayyan.

51.. Voir par exemple la biographie d'Abu al-Hassan publiée sur le forum Internet djihadiste al-Hikma le 5 juin 2005.

52.. Ghantous G., « Islamist militants urge increase in Attacks During Ramadan », Reuters, 21 octobre 2004.

53.. Smith C.S., « U.S. contends campaign has cut suicide attacks », op. cit.

54.. McDonnel P.J., « Coalition gains insight into Iraq's foreign insurgents », Los Angeles Times, 9 février 2004.

55.. Chehab Z., Inside the Resistance, New York, Nation, 2005, pp. 33-67.

56.. Hashim A.S., Insurgency and Counter-Insurgency in Iraq, Ithaca, Cornell University Press, 2006, p. 150 ; Gordon L., « Iraqi insurgents turn against "out of control" Saudi alQaeda fighters », Daily Telegraph, 30 mai 2004.

57.. Il y a bien sûr des exceptions : voir par exemple al-Saadi S., « Saudi bomb mastermind killed », Arab News, 28 août 2005.

58.. Ce sont des Saoudiens qui ont opéré l'attaque à l'intérieur d'un camp militaire américain à Mossoul en décembre 2004.

59.. Abdul Ghafour P.K., "Saudi explains how Al-Qaeda used him for terror operation ", Arab News, 18 septembre 2005.

60.. al-Dhaidi, « al-irhabiyyun fi'l-'iraq yuridun al-sa'udi imma intihariyyan aw mumawwilan... faqat ", op. cit.

61.. Whitlock C., « Saudis facing return of radicals », Washington Post, 11 juillet 2004

\section{RÉSUMÉS}

Dans cet article, Thomas Hegghammer analyse, au travers d'un échantillon de deux cent cinq «martyrs» saoudiens tués en Irak depuis 2003, les différentes trajectoires ayant conduit de jeunes Saoudiens établis d'embrasser le combat et une mort probable sur le champ de bataille irakien. L'étude de leurs trajectoires individuelles et collectives permet d'avoir une image plus complexe et détaillée de leurs antécédents, de leurs motivations, de leurs modes de recrutement ainsi que de leur rôle dans l'insurrection irakienne. En montrant que leur origine géographique 
et sociale ne joue pas un rôle déterminant, que des motivations plus généralement sociales et politiques jouent un rôle crucial et qu'il s'agit d'une génération différente de celle des «Arabes afghans » et de l'ancien réseau Al-Qaïda, cet article remet en question nombre de représentations communes du phénomène. Il montre également que, contrairement à ce qui est généralement dit, le « contingent » saoudien ne semble pas jouer un rôle crucial dans l'insurrection en Irak.

In this article Thomas Hegghammer analyses, through a sample of 205 Saudi "martyrs" killed in Iraq since 2003, the different paths having led young established Saudis to leave their life in Saudi Arabia to embrace combat and a likely death on the battlefield of Iraq. The study of their individual and collective trajectories allows getting a more complex and informed picture of their backgrounds, motivations, recruitment patterns and roles in the Iraqi insurgency. By showing that the geographical and social background of the individuals involved doesn't play a central role in their engagement, that social and political motivation are determining and that their rather young age proves them not to be former "Afghan Arabs", nor members of the old alQaida network, this article questions many common assumptions related to this phenomenon. It also shows that, as opposed to what is often said, the Saudi "contingent" does not seem to play a prominent role in the Iraqi insurgency.

\section{INDEX}

Index chronologique : 2000 - 2010

Thèmes : Al Qaïda

Mots-clés : attentat, guérillas et organisations clandestines, kamikaze (missions suicide), réseaux transnationaux

Index géographique : Irak, Moyen-Orient

\section{AUTEURS}

\section{THOMAS HEGGHAMMER}

Thomas Hegghammer est chercheur au Centre norvégien de recherche pour la défense et doctorant à l'Institut d'études politiques de Paris. Ses recherches portent sur le militantisme islamiste en Arabie Saoudite. Il a co-écrit «Al-Qaïda dans le texte » (Paris, PUF, 2005) et a écrit un rapport pour International Crisis Group, et des articles pour des revues telles que l'International Journal of Middle East Studies, the Middle East Journal et Middle East Policy. 